\title{
A facile one-pot method for preparation of succinic esters containing several functional groups
}

\author{
Zahra Hassani, Mohammad Reza Islami*, Hassan Sheibani, Maryam Kalantari, \\ and Kazem Saidi
}

Department of Chemistry, Shahid Bahonar Uuniversity of Kerman, Kerman 76169, Iran

E-mail: mrislami@mail.uk.ac.ir

\begin{abstract}
Hydrazine derivatives undergo reactions with acetylenic esters in the presence of triphenylphosphine yielding multiple functionalized succinic ester derivatives in good to excellent yields.
\end{abstract}

Keywords: Diacetyl hydrazine, acetyl hydrazine, succinic esters, triphenylphosphine

\section{Introduction}

The synthesis and chemistry of hydrazine derivatives have been attracted considerable attention due to their pharmaceutical and biological properties ${ }^{1}$. These compounds are an interesting class of materials that have been used as building blocks in heterocyclic systems containing N-N bonds $^{2}$ and azapeptides ${ }^{3}$. Some hydrazines such as phthalazin-1-yl-hydrazine were widely used as a general antihypertensive, and vasodilator agents, some years ago and at the present time as a first-line drug in the management of pregnancy-induced hypertension ${ }^{4,5}$. On the other hand, most of the hydrazines are endothermic systems and they are known to decompose easily in the presence of reactive intermediate such as free radicals which were commonly used as rocket fuels ${ }^{6,7}$.

In resent years, the synthesis of hydrazines connected to phosphorus atom has drawn much more attention. A series of these compounds have been reported in the literature which were obtained from multi-step procedures ${ }^{8,9}$. Since the synthetic application of the compounds has increased enormously in medicinal chemistry we now wish to report a one-step method for the synthesis of succinic esters containing hydrazine group along with other functional groups by reaction of dialkyl acetylenedicarboxylate with hydrazine derivatives in the presence of a desired phosphine such as triphenylphosphine as a good nucleophile. 


\section{Results and Discussion}

The results presented here are based on the proposed sequence below (Scheme1). It is known that the reaction of acetylenic esters and $\mathrm{Ph}_{3} \mathrm{P}$ produces the carbene-ylide intermediate 2 which is sufficiently stabilized by resonance ${ }^{10,11}$. Thus, compounds $\mathbf{4 a - d}$ are apparently obtained from the initial addition of triphenylphosphine as a good nucleophile ${ }^{12-16}$ to acetylenic esters as a Michael acceptor ${ }^{17}$ and concomitant protonation of the carbene-ylide intermediate 2 by the NHacid. Then positively charged ion is attacked by the nitrogen of the conjugated base of the NHacid or $\mathrm{NH}_{2}$ group to form succinic esters containing several functional groups 4 . It should be noted that in the final step of the reaction of compound $\mathbf{3 b}$ with positive charge, the $\mathrm{NH}_{2}$ group act as a stronger nucleophile than the conjugated base of the amide group.

The structures of compounds 4a-d were characterized on the basis of their elemental analyses and their IR, mass, high-field ${ }^{1} \mathrm{H}$ NMR and ${ }^{13} \mathrm{C}$ NMR spectra. The nature of these compounds as 1:1:1 adducts were apparent from the corresponding mass spectra, which displayed fairly weak molecular ion peaks at $\mathrm{m} / \mathrm{z} 520,548,478$ and 506 for succinic esters derivatives 4a-d, respectively. The initial fragmentation involves the loss of the $\mathrm{PPh}_{3}$ and ester moieties. The ${ }^{1} \mathrm{H}$ NMR and ${ }^{13} \mathrm{C}$ NMR spectral data of new succinic esters 4a-d exhibited a mixture of two conformational isomers. The ylide moiety of these compounds is strongly conjugated with the adjacent carbonyl group and rotation about the partial double bond in 4-(E), 4-(Z) geometrical isomer is low on the NMR time scale at ambient temperature (Scheme 1). Conformational isomers in phosphoranes have been previously established and reported in the literature ${ }^{18-20}$.

The ${ }^{1} \mathrm{H}$ NMR spectra of $\mathbf{4 a}$ showed four sharp signals due to methyl protons of acetyl groups at $\delta=1.95,1.97,2.00$ and $2.04 \mathrm{ppm}$ along with four sharp lines at $\delta=3.18,3.55,3.72$ and $3.78 \mathrm{ppm}$ arising from methoxy protons. Methine protons appeared as two doublet peaks at $\delta=5.09$ and $5.13 \mathrm{ppm}$ respectively for the $\mathrm{Z}$ and $\mathrm{E}$ geometrical isomrs and a fairly broad signals at $\delta=8.19 \mathrm{ppm}$, for the NH proton. The aromatic protons appeared as a multiplet at $\mathrm{d}=7.47$ $7.73 \mathrm{ppm}$. The ${ }^{13} \mathrm{C}$ NMR spectrum of $4 \mathbf{a}$ displayed twenty four distinct resonances in agreement with the mixture of two conformational isomers. Although the presence of the ${ }^{31} \mathrm{P}$ nucleus has complicated both the ${ }^{1} \mathrm{H}$ and ${ }^{13} \mathrm{C}$ NMR spectra of $\mathbf{4 a}$, it helps in assignment of signals by longrange spin-spin couplings with ${ }^{1} \mathrm{H}$ and ${ }^{13} \mathrm{C}$ nuclei. 


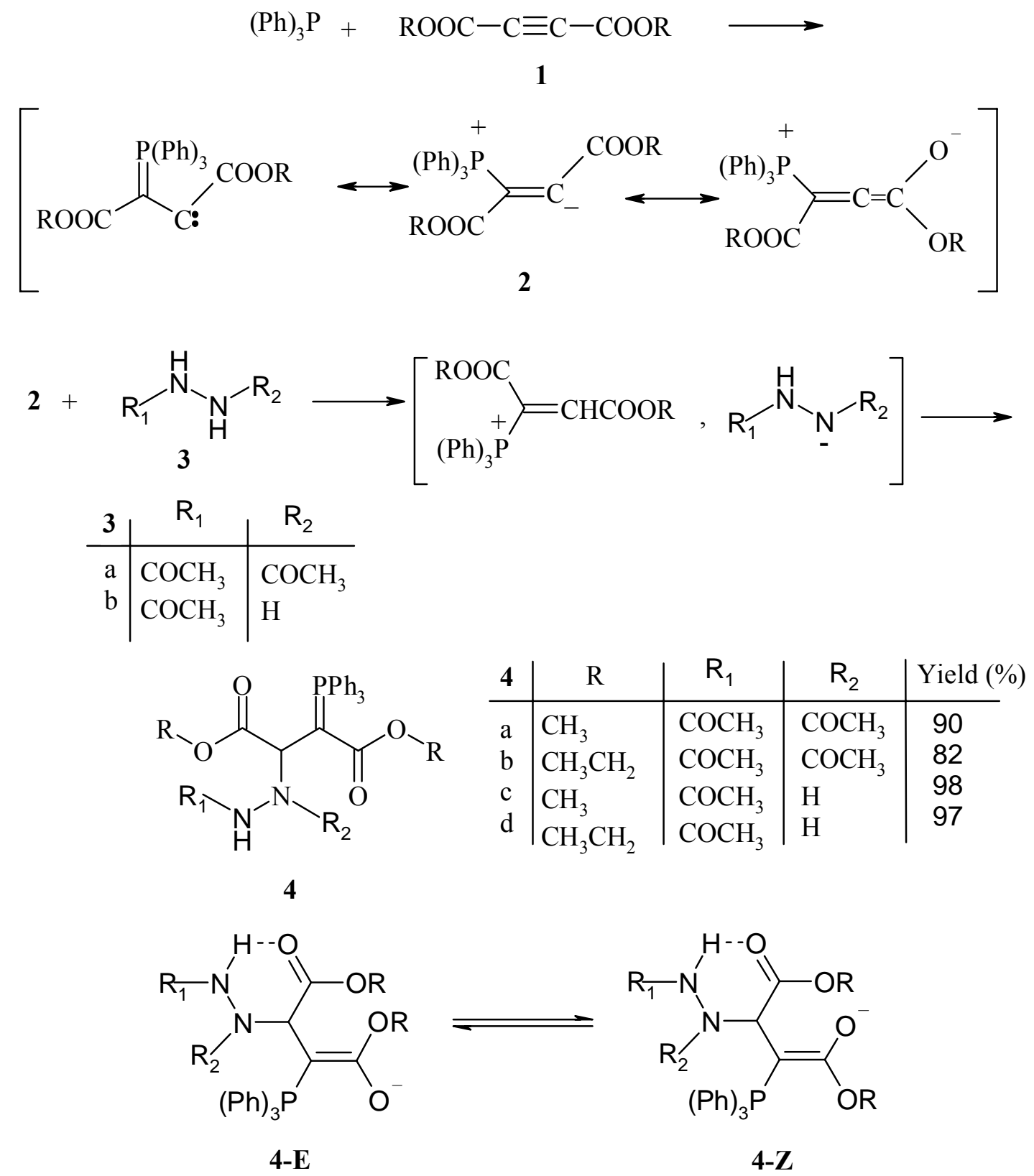

\section{Scheme 1}

The ${ }^{1} \mathrm{H}$ and ${ }^{13} \mathrm{C}$ NMR spectra of compound $\mathbf{4 b}$ are similar to those of $\mathbf{4 a}$, except for the signals from the ester group, which appear as characteristic resonance lines with the corresponding chemical shifts. The ${ }^{1} \mathrm{H}$ and ${ }^{13} \mathrm{C}$ NMR spectroscopic data for compounds $4 \mathbf{c}-\mathbf{d}$ are also consistent with the proposed structures. The structural assignments made for succinic esters derivatives $4 \mathbf{a}-\mathbf{d}$ on the basis of the ${ }^{1} \mathrm{H}$ and ${ }^{13} \mathrm{C}$ NMR spectra were supported by their IR spectra. In the IR spectra of $\mathbf{4 a - b}$ an absorption band at $3354 \mathrm{~cm}^{-1}$ were detected, due to the NH group. The IR spectra of compounds $\mathbf{4 c - d}$ are similar to those of compounds $\mathbf{4 a - b}$, except for the new absorption band from the other NH group, which appears at $3304 \mathrm{~cm}^{-1}$. The carbonyl 
region of the spectra exhibited absorption bands for each compound. Of special interest is the ester absorption at $1765-1617 \mathrm{~cm}^{-1}$ for these compounds. Conjugation with negative charge accounts for the reduction of the wave numbers of the carbonyl absorption bands.

\section{Experimental Section}

General Procedures. Dialkyl acetylenedicarboxylate, diacetyl hydrazine, acetyl hydrazine and triphenylphosphine were obtained from Fluka Chemical Co. and were used without further purification. Melting points were obtained on a Gallenkamp melting point apparatus and are uncorrected. Elemental analyses for $\mathrm{C}, \mathrm{H}$ and $\mathrm{N}$ were performed by University of Ferdosi using a Heracus CHN-O-Rapid analyzer. IR spectra were measured on a Mattson 1000 FT-IR spectrometer. ${ }^{1} \mathrm{H}$ and ${ }^{13} \mathrm{C}$ NMR spectra were recorded on a BRUKER DRX-500 AVANCE spectrometer at 500 and $125.77 \mathrm{MHz}$, respectively. Mass spectra were recorded on a shimadzo MS-QP2000A mass spectrometer operating at an ionization potentional of $70 \mathrm{eV}$.

Dimethyl 2-(2-acetylhydrazino)-3-(1,1,1-triphenyl- $\lambda^{\mathbf{5}}$-phosphanylidene) succinate (4a). At ambient temperature $(0.24 \mathrm{ml}, 2 \mathrm{mmol})$ of dimethyl acetylenedicarboxylate was added dropwise to a stirred emulsion of $(0.53 \mathrm{~g}, 2 \mathrm{mmol})$ of triphenylphosphine and $(0.232 \mathrm{~g}, 2 \mathrm{mmol})$ of diacetyl hydrazine in a $9 \mathrm{~mL}$ mixture of ethyl acetate-hexane (6:3). After the addition was complete (approximately 5 minutes) the mixture was stirred for another $30 \mathrm{~min}$ and filtered. The solid collected in the filter was washed thoroughly with ethyl acetate to give a white powder. ( $0.94 \mathrm{~g}$, m.p 167-169 ${ }^{\circ}$ C. yield $\left.90 \%\right)$; IR (KBr) $\left(v_{\max }, \mathrm{cm}^{-1}\right)$ : 1765, 1741, 1716 and 1691(C=O). MS, $\mathrm{m} / z$ (\%): $520\left(\mathrm{M}^{+}, 3\right), 489$ (5), 405 (98), 277 (33), 262 (99), 183 (95), 43 (100) Anal. Calcd. for $\mathrm{C}_{28} \mathrm{H}_{29} \mathrm{~N}_{2} \mathrm{O}_{6} \mathrm{P}$ (520.53): C, 64.61; H, 5.62; N, 5.38 \%. Found: C, 64.38; H, 5.32; N, 5.52 \%.

Major conformational isomer 4a-(Z) (75\%), ${ }^{1} \mathrm{H}$ NMR: $\delta 1.97$ and $2.04\left(6 \mathrm{H}, 2 \mathrm{~s}, 2 \mathrm{CH}_{3}\right)$, 3.18 and $3.78\left(6 \mathrm{H}, 2 \mathrm{~s}, 2 \mathrm{OCH}_{3}\right), 5.09\left(1 \mathrm{H}, \mathrm{d},{ }^{3} \mathrm{~J}_{\mathrm{PH}} 18.1 \mathrm{~Hz}, \mathrm{P}=\mathrm{C}-\mathrm{CH}\right), 7.47-7.73(30 \mathrm{H}, \mathrm{m} \text {, arom })^{*}$ (* - For two conformational isomers), $8.19(1 \mathrm{H}$, brod band, $\mathrm{NH}){ }^{*} .{ }^{13} \mathrm{C} \mathrm{NMR}: \delta 21.03$ and 21.07 $\left(2 \mathrm{CH}_{3}\right), 40.69\left(\mathrm{~d},{ }^{1} \mathrm{~J}_{\mathrm{PC}} 126.1 \mathrm{~Hz}, \mathrm{P}=\mathrm{C}\right), 49.38$ and $52.54\left(2 \mathrm{OCH}_{3}\right), 59.04\left(\mathrm{~d},{ }^{2} \mathrm{~J}_{\mathrm{PC}} 17.4 \mathrm{~Hz}, \mathrm{P}=\mathrm{C}-\right.$ $\mathrm{CH}), 126.03\left(\mathrm{~d},{ }^{1} \mathrm{~J}_{\mathrm{PC}} 91.9 \mathrm{~Hz}, \mathrm{C}^{\text {ipso }}\right), 128.65\left(\mathrm{~d},{ }^{3} \mathrm{~J}_{\mathrm{PC}} 12.3 \mathrm{~Hz}, \mathrm{C}^{\text {meta }}\right), 132.22\left(\mathrm{C}^{\text {para }}\right)^{*}, 133.77(\mathrm{~d}$, $\left.{ }^{2} \mathrm{~J}_{\mathrm{PC}} 10.1 \mathrm{~Hz}, \mathrm{C}^{\text {ortho }}\right), 170.74$ and $172.43(2 \mathrm{C}=\mathrm{O}), 171.42\left(\mathrm{~d},{ }^{2} \mathrm{~J}_{\mathrm{PC}} 12.8 \mathrm{~Hz}, \mathrm{C}=\mathrm{O}\right){ }^{*}, 172.61\left(\mathrm{~d},{ }^{3} \mathrm{~J}_{\mathrm{PC}}\right.$ $12.0 \mathrm{~Hz}, \mathrm{C}=\mathrm{O})^{*}$.Minor conformational isomer 4a-(E) $(24.4 \%),{ }^{1} \mathrm{H}$ NMR: $\delta 1.95$ and $2.00(6 \mathrm{H}, 2 \mathrm{~s}$, $\left.2 \mathrm{CH}_{3}\right), 3.55$ and $3.72\left(6 \mathrm{H}, 2 \mathrm{~s}, 2 \mathrm{OCH}_{3}\right), 5.13\left(1 \mathrm{H}, \mathrm{d},{ }^{3} \mathrm{~J}_{\mathrm{PH}} 20.1 \mathrm{~Hz}, \mathrm{P}=\mathrm{C}-\mathrm{CH}\right) .{ }^{13} \mathrm{C}$ NMR: $\delta 20.91$ and $21.07\left(2 \mathrm{CH}_{3}\right), 41.10\left(\mathrm{~d},{ }^{1} \mathrm{~J}_{\mathrm{PC}} 133.4 \mathrm{~Hz}, \mathrm{P}=\mathrm{C}\right), 50.11$ and $52.52\left(2 \mathrm{OCH}_{3}\right), 58.78\left(\mathrm{~d},{ }^{2} \mathrm{~J}_{\mathrm{PC}} 17.03\right.$ $\mathrm{Hz}, \mathrm{P}=\mathrm{C}-\mathrm{CH}), 125.69$ (d, $\left.{ }^{1} \mathrm{~J}_{\mathrm{PC}} 92.3 \mathrm{~Hz}, \mathrm{C}^{\mathrm{ipso}}\right), 133.86\left(\mathrm{~d},{ }^{2} \mathrm{~J}_{\mathrm{PC}} 9.8 \mathrm{~Hz}, \mathrm{C}^{\text {ortho }}\right), 169.83$ and 172.78 $(2 \mathrm{C}=\mathrm{O})$.

Diethyl 2-(2-acetylhydrazino)-3-(1,1,1-triphenyl- $\lambda^{\mathbf{5}}$-phosphanylidene) succinate (4b). (0.9 g, m.p 106-109 ${ }^{\circ}$ C. yield 82\%); IR (KBr) $\left(v_{\max }, \mathrm{cm}^{-1}\right)$ : 1765, 1742, 1716 and $1693(\mathrm{C}=\mathrm{O}) . \mathrm{MS}, \mathrm{m} / \mathrm{z}$ (\%): $548\left(\mathrm{M}^{+}, 11\right), 434$ (18), 461 (24), 277 (20), 262 (10), 183 (9), 86 (77), 49 (100). Anal. Calcd. for $\mathrm{C}_{30} \mathrm{H}_{33} \mathrm{~N}_{2} \mathrm{O}_{6} \mathrm{P}$ (548.58): C, 65.68; H, 6.06; N, 5.11 \%. Found: C, 65.30; H, 5.99; N, $5.50 \%$. 
Major conformational isomer 4b-(Z) (72.7\%), ${ }^{1} \mathrm{H}$ NMR: $\delta 0.54\left(3 \mathrm{H}, \mathrm{t},{ }^{3} \mathrm{~J}_{\mathrm{HH}} 7.0 \mathrm{~Hz}, \mathrm{CH}_{3}\right)$, $1.31\left(3 \mathrm{H}, \mathrm{t},{ }^{3} \mathrm{~J}_{\mathrm{HH}} 7.1 \mathrm{~Hz}, \mathrm{CH}_{3}\right), 2.03$ and $2.04\left(6 \mathrm{H}, 2 \mathrm{~s}, 2 \mathrm{CH}_{3}\right), 3.96-4.31\left(8 \mathrm{H}, \mathrm{m}, 2 \mathrm{CH}_{2}\right)^{*}, 5.10(1 \mathrm{H}$, d, $\left.{ }^{3} \mathrm{~J}_{\mathrm{PH}} 18.2 \mathrm{~Hz}, \mathrm{P}=\mathrm{C}-\mathrm{CH}\right), 7.44-7.75(30 \mathrm{H}, \mathrm{m} \text {, arom })^{*}, 8.23(1 \mathrm{H}$, brod band, $\mathrm{NH}){ }^{*} \cdot{ }^{13} \mathrm{C}$ NMR: $\delta$ 14.17 and $14.14\left(2 \mathrm{CH}_{3}\right), 21.01$ and $21.03\left(2 \mathrm{CH}_{3}\right), 4.47\left(\mathrm{~d},{ }^{1} \mathrm{~J}_{\mathrm{PC}} 125.9 \mathrm{~Hz}, \mathrm{P}=\mathrm{C}\right), 58.01$ and 61.29 $\left(2 \mathrm{OCH}_{2}\right), 59.05\left(\mathrm{~d},{ }^{2} \mathrm{~J}_{\mathrm{PC}} 17.6 \mathrm{~Hz}, \mathrm{P}=\mathrm{C}-\mathrm{CH}\right), 126.27\left(\mathrm{~d},{ }^{1} \mathrm{~J}_{\mathrm{PC}} 91.8 \mathrm{~Hz}, \mathrm{C}^{\mathrm{ipso}}\right), 128.56\left(\mathrm{~d},{ }^{3} \mathrm{~J}_{\mathrm{PC}} 12.3\right.$ $\left.\mathrm{Hz}, \mathrm{C}^{\text {meta }}\right)^{*}, 131.98\left(\mathrm{C}^{\text {para }}\right)^{*}, 133.84\left(\mathrm{~d},{ }^{2} \mathrm{~J}_{\mathrm{PC}} 8.4 \mathrm{~Hz}, \mathrm{C}^{\text {ortho }}\right)^{*}, 170.70$ and $172.47(2 \mathrm{C}=\mathrm{O}), 170.94(\mathrm{~d}$, $\left.{ }^{2} \mathrm{~J}_{\mathrm{PC}} 13.1 \mathrm{~Hz}, 2 \mathrm{C}=\mathrm{O}\right)^{*}, 172.05\left(\mathrm{~d},{ }^{3} \mathrm{~J}_{\mathrm{PC}} 12.0 \mathrm{~Hz}, 2 \mathrm{C}=\mathrm{O}\right){ }^{*}$. Minor conformational isomer $4 \mathbf{b}-(\mathrm{E})$ (27.3\%), ${ }^{1} \mathrm{H}$ NMR: $\delta 1.21\left(3 \mathrm{H}, \mathrm{t},{ }^{3} \mathrm{~J}_{\mathrm{HH}} 7.1 \mathrm{~Hz}, \mathrm{CH}_{3}\right), 1.31\left(3 \mathrm{H}, \mathrm{t},{ }^{3} \mathrm{~J}_{\mathrm{HH}} 7.0 \mathrm{~Hz}, \mathrm{CH}_{3}\right), 1.97$ and 2.07 $\left(6 \mathrm{H}, 2 \mathrm{~s}, 2 \mathrm{CH}_{3}\right), 5.13\left(1 \mathrm{H}, \mathrm{d},{ }^{3} \mathrm{~J}_{\mathrm{PH}} 19.4 \mathrm{~Hz}, \mathrm{P}=\mathrm{C}-\mathrm{CH}\right) .{ }^{13} \mathrm{C}$ NMR: $\delta 14.25$ and $14.48\left(2 \mathrm{CH}_{3}\right), 21.12$ and $21.14\left(2 \mathrm{CH}_{3}\right), 40.97\left(\mathrm{~d},{ }^{1} \mathrm{~J}_{\mathrm{PC}} 139.1 \mathrm{~Hz}, \mathrm{P}=\mathrm{C}\right), 58.74\left(\mathrm{~d},{ }^{2} \mathrm{~J}_{\mathrm{PC}} 17.5 \mathrm{~Hz}, \mathrm{P}=\mathrm{C}-\mathrm{CH}\right), 61.05$ and $61.32\left(2 \mathrm{OCH}_{2}\right), 125.86\left(\mathrm{~d},{ }^{1} \mathrm{~J}_{\mathrm{PC}} 92.3 \mathrm{~Hz}, \mathrm{C}^{\mathrm{ipso}}\right), 169.86$ and $172.35(2 \mathrm{C}=\mathrm{O})$.

Dimethyl 2-(1-acetylhydrazino)-3-(1,1,1-triphenyl- $\lambda^{\mathbf{5}}$-phosphanylidene) succinate (4c). $(0.94$ g, m.p $160-162^{\circ} \mathrm{C}$. yield $98 \%$ ); IR $(\mathrm{KBr})\left(v_{\max }, \mathrm{cm}^{-1}\right): 1741,1716$ and $1691(\mathrm{C}=\mathrm{O}) . \mathrm{MS}, \mathrm{m} / z(\%)$ : $478\left(\mathrm{M}^{+}, 3\right), 461$ (22), 434 (22), 419 (38), 277 (24), 262 (10), 183 (12), 43 (100). Anal. Calcd. for $\mathrm{C}_{26} \mathrm{H}_{27} \mathrm{~N}_{2} \mathrm{O}_{5} \mathrm{P}$ (478.49): C, 65.27; H, 5.69; N, 5.85 \%. Found: C, 65.20; H, 5.50; N, 6.12 \%.

Major conformational isomer $4 \mathrm{c}-(\mathrm{Z})(54 \%),{ }^{1} \mathrm{H} \mathrm{NMR}: \delta 1.74\left(3 \mathrm{H}, \mathrm{s}, \mathrm{CH}_{3}\right), 3.11$ and 3.74 $\left(6 \mathrm{H}, 2 \mathrm{~s}, 2 \mathrm{OCH}_{3}\right), 5.25\left(1 \mathrm{H}, \mathrm{d},{ }^{3} \mathrm{~J}_{\mathrm{PH}} 10.5 \mathrm{~Hz}, \mathrm{P}=\mathrm{C}-\mathrm{CH}\right), 5.20$ and $7.29(2 \mathrm{H} \text {, brod band, } 2 \mathrm{NH})^{*}$. 7.49-7.75 (30H, m, arom) ${ }^{*} .{ }^{13} \mathrm{C}$ NMR: $\delta 19.34\left(\mathrm{CH}_{3}\right), 41.26\left(\mathrm{~d},{ }^{1} \mathrm{~J}_{\mathrm{PC}} 126.8 \mathrm{~Hz}, \mathrm{P}=\mathrm{C}\right), 49.07$ and $52.32\left(2 \mathrm{OCH}_{3}\right), 62.93\left(\mathrm{~d},{ }^{2} \mathrm{~J}_{\mathrm{PC}} 14.6 \mathrm{~Hz}, \mathrm{P}=\mathrm{C}-\mathrm{CH}\right), 126.63\left(\mathrm{~d},{ }^{1} \mathrm{~J}_{\mathrm{PC}} 92.3 \mathrm{~Hz}, \mathrm{C}^{\mathrm{ipso}}\right), 128.83\left(\mathrm{~d},{ }^{3} \mathrm{~J}_{\mathrm{PC}}\right.$ $\left.13.1 \mathrm{~Hz}, \mathrm{C}^{\text {meta }}\right), 132.29\left(\mathrm{C}^{\text {para }}\right)^{*}, 133.71\left(\mathrm{~d},{ }^{2} \mathrm{~J}_{\mathrm{PC}} 9.0 \mathrm{~Hz}, \mathrm{C}^{\text {ortho }}\right), 170.07\left(\mathrm{~d},{ }^{2} \mathrm{~J}_{\mathrm{PC}} 13.2 \mathrm{~Hz}, \mathrm{C}=\mathrm{O}\right)$, $174.48\left(\mathrm{~d},{ }^{3} \mathrm{~J}_{\mathrm{PC}} 9.5 \mathrm{~Hz}, \mathrm{C}=\mathrm{O}\right), 170.07(2 \mathrm{C}=\mathrm{O})$. Minor conformational isomer $4 \mathrm{c}-(\mathrm{E})(46 \%),{ }^{1} \mathrm{H}$ NMR: $\delta 1.80\left(3 \mathrm{H}, \mathrm{s}, \mathrm{CH}_{3}\right), 3.53$ and $3.72\left(6 \mathrm{H}, 2 \mathrm{~s}, 2 \mathrm{OCH}_{3}\right), 4.84\left(1 \mathrm{H}, \mathrm{d},{ }^{3} \mathrm{~J}_{\mathrm{PH}} 10.0 \mathrm{~Hz}, \mathrm{P}=\mathrm{C}-\mathrm{CH}\right)$. ${ }^{13} \mathrm{C}$ NMR: $\delta 21.42\left(\mathrm{CH}_{3}\right), 41.49\left(\mathrm{~d},{ }^{1} \mathrm{~J}_{\mathrm{PC}} 127.4 \mathrm{~Hz}, \mathrm{P}=\mathrm{C}\right), 50.07$ and $50.21\left(2 \mathrm{OCH}_{3}\right), 63.44(\mathrm{~d}$, $\left.{ }^{2} \mathrm{~J}_{\mathrm{PC}} 14.2 \mathrm{~Hz}, \mathrm{P}=\mathrm{C}-\mathrm{CH}\right), 126.32\left(\mathrm{~d},{ }^{1} \mathrm{~J}_{\mathrm{PC}} 92.8 \mathrm{~Hz}, \mathrm{C}^{\text {ipso }}\right), 128.72\left(\mathrm{~d},{ }^{3} \mathrm{~J}_{\mathrm{PC}} 14.6 \mathrm{~Hz}, \mathrm{C}^{\text {meta }}\right), 132.03$ $\left(\mathrm{C}^{\text {para }}\right), 169.77\left(\mathrm{~d},{ }^{2} \mathrm{~J}_{\mathrm{PC}} 12.6 \mathrm{~Hz}, \mathrm{C}=\mathrm{O}\right), 172.35(\mathrm{C}=\mathrm{O}), 175.12\left(\mathrm{~d},{ }^{3} \mathrm{~J}_{\mathrm{PC}} 10.0 \mathrm{~Hz}, \mathrm{C}=\mathrm{O}\right)$.

Diethyl 2-(1-acetylhydrazino)-3-(1,1,1-triphenyl- $\lambda^{\mathbf{5}}$-phosphanylidene) succinate (4d). (0.98 g, m.p 154-156 C. yield 97\%); IR (KBr) $\left(v_{\max }, \mathrm{cm}^{-1}\right): 1741,1716$ and $1691(\mathrm{C}=\mathrm{O}) . \mathrm{MS}, \mathrm{m} / z(\%)$ : 506 ( $\left.\mathrm{M}^{+}, 6\right), 461$ (4), 434 (2), 277 (100), 262 (61), 183 (91), 73 (38). Anal. Calcd. for $\mathrm{C}_{28} \mathrm{H}_{31} \mathrm{~N}_{2} \mathrm{O}_{5} \mathrm{P}$ (506.54): C, 66.39; H, 6.12; N, $5.53 \%$. Found: C, 66.31; H, 6.30; N, $5.63 \%$.

Major conformational isomer 4d-(Z) (55.5\%), ${ }^{1} \mathrm{H}$ NMR: $\delta 0.44\left(3 \mathrm{H}, \mathrm{t},{ }^{3} \mathrm{~J}_{\mathrm{HH}} 7.0 \mathrm{~Hz}, \mathrm{CH}_{3}\right)$, $1.31\left(3 \mathrm{H}, \mathrm{t},{ }^{3} \mathrm{~J}_{\mathrm{HH}} 6.8 \mathrm{~Hz}, \mathrm{CH}_{3}\right), 1.81\left(6 \mathrm{H}, 2 \mathrm{~s}, 2 \mathrm{CH}_{3}\right), 3.14-4.29\left(8 \mathrm{H}, \mathrm{m}, 2 \mathrm{OCH}_{2}\right)^{*}, 5.28\left(1 \mathrm{H}, \mathrm{d},{ }^{3} \mathrm{~J}_{\mathrm{PH}}\right.$ $10.4 \mathrm{~Hz}, \mathrm{P}=\mathrm{C}-\mathrm{CH}), 7.28$ and $7.32(2 \mathrm{H} \text {, brod band, } 2 \mathrm{NH})^{*}, 7.49-7.77(30 \mathrm{H}, \mathrm{m} \text {, arom })^{*} .{ }^{13} \mathrm{C} \mathrm{NMR}$ : $\delta 13.89$ and $14.31\left(2 \mathrm{CH}_{3}\right), 19.35\left(\mathrm{CH}_{3}\right), 41.08\left(\mathrm{~d},{ }^{1} \mathrm{~J}_{\mathrm{PC}} 127.1 \mathrm{~Hz}, \mathrm{P}=\mathrm{C}\right), 57.68$ and 60.83 (2 $\left.\mathrm{OCH}_{2}\right), 63.25\left(\mathrm{~d},{ }^{2} \mathrm{~J}_{\mathrm{PC}} 15.3 \mathrm{~Hz}, \mathrm{P}=\mathrm{C}-\mathrm{CH}\right), 126.84\left(\mathrm{~d},{ }^{1} \mathrm{~J}_{\mathrm{PC}} 92.2 \mathrm{~Hz}, \mathrm{C}^{\mathrm{ipso}}\right), 128.73\left(\mathrm{~d},{ }^{3} \mathrm{~J}_{\mathrm{PC}} 12.3 \mathrm{~Hz}\right.$, $\left.\mathrm{C}^{\text {meta }}\right)^{*}, 132.28\left(\mathrm{C}^{\text {para }}\right)^{*}, 133.73\left(\mathrm{~d},{ }^{2} \mathrm{~J}_{\mathrm{PC}} 9.7 \mathrm{~Hz}, \mathrm{C}^{\text {ortho }}\right), 169.35\left(\mathrm{~d},{ }^{2} \mathrm{~J}_{\mathrm{PC}} 12.8 \mathrm{~Hz}, \mathrm{C}=\mathrm{O}\right), 174.03(\mathrm{~d}$, $\left.{ }^{3} \mathrm{~J}_{\mathrm{PC}} 10.1 \mathrm{~Hz}, \mathrm{C}=\mathrm{O}\right), 175.02(\mathrm{C}=\mathrm{O})$. Minor conformational isomer 4d-(E) $(44.5 \%),{ }^{1} \mathrm{H} \mathrm{NMR}: \delta$ $1.17\left(3 \mathrm{H}, \mathrm{t},{ }^{3} \mathrm{~J}_{\mathrm{HH}} 6.2 \mathrm{~Hz}, \mathrm{CH}_{3}\right), 1.26\left(3 \mathrm{H}, \mathrm{t},{ }^{3} \mathrm{~J}_{\mathrm{HH}} 7.0 \mathrm{~Hz}, \mathrm{CH}_{3}\right), 1.76\left(3 \mathrm{H}, \mathrm{s}, \mathrm{CH}_{3}\right), 4.86(1 \mathrm{H}, \mathrm{d}$, $\left.{ }^{3} \mathrm{~J}_{\mathrm{PH}} 10.2 \mathrm{~Hz}, \mathrm{P}=\mathrm{C}-\mathrm{CH}\right) .{ }^{13} \mathrm{C} \mathrm{NMR}: \delta 14.01$ and $14.98\left(2 \mathrm{CH}_{3}\right), 21.49\left(\mathrm{CH}_{3}\right), 41.36\left(\mathrm{~d},{ }^{1} \mathrm{~J}_{\mathrm{PC}} 127.1\right.$ $\mathrm{Hz}, \mathrm{P}=\mathrm{C}), 58.31$ and $58.10\left(2 \mathrm{OCH}_{2}\right), 62.79\left(\mathrm{~d},{ }^{2} \mathrm{~J}_{\mathrm{PC}} 14.0 \mathrm{~Hz}, \mathrm{P}=\mathrm{C}-\mathrm{CH}\right), 127.04\left(\mathrm{~d},{ }^{1} \mathrm{~J}_{\mathrm{PC}} 92.4 \mathrm{~Hz}\right.$, 
$\left.\mathrm{C}^{\text {ipso }}\right), 128.62\left(\mathrm{~d},{ }^{3} \mathrm{~J}_{\mathrm{PC}} 15.3 \mathrm{~Hz}, \mathrm{C}^{\text {meta }}\right), 131.98\left(\mathrm{C}^{\text {para }}\right), 133.87\left(\mathrm{~d},{ }^{2} \mathrm{~J}_{\mathrm{PC}} 9.8 \mathrm{~Hz}, \mathrm{C}^{\text {ortho }}\right), 169.74\left(\mathrm{~d},{ }^{2} \mathrm{~J}_{\mathrm{PC}}\right.$ $12.5 \mathrm{~Hz}, \mathrm{C}=\mathrm{O}), 174.90\left(\mathrm{~d},{ }^{3} \mathrm{~J}_{\mathrm{PC}} 10.0 \mathrm{~Hz}, \mathrm{C}=\mathrm{O}\right), 175.00(\mathrm{C}=\mathrm{O})$.

\section{Acknowledgements}

The authors express appreciation to the Shahid Bahonar University of Kerman Faculty Research Committee for its support of this investigation.

\section{References}

1. Miyadera, T. The Chemistry of hydrazo Aza and Azoxy Groups; Patai, S., Ed.; John Wiley: London, Part.1, 1975, p 495.

2. Schiessl, H. Aldrichimica Acta. 1980, 13, 33.

3. Galite, J. Synthesis. 1989, 405.

4. Powers, D. R.; Papadakos, P. J.; Willin, J. D. J. Emerg. Med. 1998, 16, 191.

5. Vidrio, H.; Fernandez, G.; Medina, M.; Alvarez, E.; Orallo, F. Vascular Pharmacol. 2003, $40,13$.

6. Harris, G. W.; Atkinson, R.; Pitts, J. N., J. Phys. Chem. 1997, 83, 2557.

7. Armatrong, D. A.; Yu, D.; Rauk, A. J. Phys. Chem. 1997, 101, 4761.

8. Barluenga, J.; Merini, I.; Vina, S.; Palacios, F. Synthesis, 1990, 398.

9. Palacios, F.; Retana, A. M. O.; Pagalday, J. Tetrahedron. 1999, 55, 14451.

10. Caesar, J.; Griffiths, D. V.; Griffiths, P. A.; Tebby, T. J. J. Chem. Soc., Perkin Trans 1, 1989, 2425.

11. Caesar, J.; Griffiths, D. V.; Griffiths, P. A.; Tebby, T. J. J. Chem. Soc,. Perkin Trans 1, 1990, 2329.

12. Engel, R. Synthesis of Carbon-Phosphorus Bonds; CRC Press: Bota Raton, FL, 1988.

13. Maryano, B. E.; Reitz, A. B. Chem. Rev. 1989, 89, 863.

14. Cherkasov, R. A.; Pudovik, M.A. Russ. Chem. Rev. 1994, 63, 1019.

15. Grayson, M.; Griffith, E. J. Topic in Phosphorus Chemistry; Interscience: New York, 1972, Vol. 7.

16. Islami, M. R.; Hassani, Z.; Sheibani, H.; Abdolahzadeh, B.; Etminan, N. Tetrahedron, 2003, 59, 4993.

17. Islami, M. R.; Mollazehi, F.; Badiei, A.; Sheibani, H. Arkivoc. 2005, (xv), 25.

18. Bestmann, H. J.; Joachim, G.; Lengyel, T.; Oth, J. F.; Merenyi, R.; Weitkamp, H. Tetrahedron. Lett. 1966, 3355.

19. Bestmann, H. J.; Snyder, J. P. J. Am. Chem. Soc. 1967, 89, 3963.

20. Hooper, D. L.; Garagan, S. J. Org. Chem., 1994, 59, 1126. 